\title{
EXPLORATION OF SPHERICAL TORUS PHYSICS IN THE NSTX DEVICE*
}

\author{
M. Ono, S. Kaye, M. Peng, G. Barnes, W. Blanchard, M. Carter ${ }^{1}$, J. Chrzanowski, \\ L. Dudek, R. Ewig², D. Gates, R. Hatcher, R. Majeski, T. Jarboe' ${ }^{2}$, S. Jardin, D. \\ Johnson, M. Kalish, R. Kaita, C. Kessel, H. Kugel, B. McCormack, R. Maingi ${ }^{1}$, J. \\ Manickam, J. Menard, D. Mueller, B. Nelson¹, B. Nelson², C. Neumeyer, G. Oliaro, \\ F. Paolletti ${ }^{3}$, R. Parsells, E. Perry, N. Pomphrey, S. Ramakrishnan, R. Raman ${ }^{2}$, G. \\ Rewoldt, J. Robinson, A.L. Roquemore, P. Ryan ${ }^{1}$, S. Sabbagh ${ }^{2}$, D. Swain ${ }^{1}$, E. \\ Synakowski, M. Viola, M. Williams, J.R. Wilson, and the NSTX Team. \\ Princeton Plasma Physics Laboratory, Princeton, NJ 08543, USA \\ 1Oak Ridge National Laboratory, Oak Ridge, TN 37831, USA \\ 2 University of Washington, Seattle, Washington, USA \\ 3 Columbia University, New York, N.Y., USA \\ *Work is supported by US Dept. of Energy contract No. DE-AC02-76CH03073.
}

\begin{abstract}
The National Spherical Torus Experiment (NSTX) is being built at PPPL to test the fusion physics principles for the ST concept at the MA level. The NSTX nominal plasma parameters are $\mathrm{R}_{0}=85 \mathrm{~cm}$, $\mathrm{a}=67 \mathrm{~cm}, \mathrm{R} / \mathrm{a} \geq 1.26, \mathrm{~B}_{\mathrm{T}}=3 \mathrm{kG}, \mathrm{I}_{\mathrm{p}}=1 \mathrm{MA}, \mathrm{q}_{95}=14$, elongation $\kappa \leq 2.2$, triangularity $\delta \leq 0.5$, and plasma pulse length of up to $5 \mathrm{sec}$. The plasma heating / current drive (CD) tools are High Harmonic Fast Wave (HHFW) (6 MW, $5 \mathrm{sec}$ ), Neutral Beam Injection (NBI) (5 MW, $80 \mathrm{keV}, 5 \mathrm{sec}$ ), and Coaxial Helicity Injection (CHI). Theoretical calculations predict that NSTX should provide exciting possibilities for exploring a number of important new physics regimes including very high plasma beta, naturally high plasma elongation, high bootstrap current fraction, absolute magnetic well, and high pressure driven sheared flow. In addition, the NSTX program plans to explore fully noninductive plasma start-up as well as a dispersive scrape-off layer for heat and particle flux handling.
\end{abstract}

\section{Motivation}

A broad range of encouraging advances has been made in the exploration of the Spherical Torus (ST) concept. ${ }^{1}$ Such advances include promising experimental data from pioneering experiments, theoretical predictions, near-term fusion energy development projections such as the Volume Neutron Source $^{2}$, and future applications such as power plant studies ${ }^{3}$. Recently, the START device has achieved a very high toroidal beta $\beta_{\mathrm{T}} \approx 40 \%$ regime with $\beta_{\mathrm{N}} \approx 5.0$ at low $\mathrm{q}_{95} \approx 3.4$ The National Spherical Torus Experiment (NSTX) is being built at PPPL to test the fusion physics principles for the ST concept at the MA level. ${ }^{5}$ The NSTX device/plasma configuration allows the plasma shaping factor, $I_{p} q_{95} / a B$, to reach as high as 80 an order of magnitude greater than that achieved in conventional high aspect ratio tokamaks. The key physics objective of NSTX is to attain an advanced ST regime; i.e., simultaneous ultra high beta $(\beta)$, high confinement, and high bootstrap current fraction $\left(\mathrm{f}_{\mathrm{bs}}\right) .{ }^{6}$ This regime is considered to be essential for the development of an economical ST power-plant because it minimizes the recirculating power and power plant core size. Other NSTX mission elements crucial for ST power plant development are the demonstration at the MA level of fully noninductive operation and the development of acceptable power and particle handling concepts.

\section{NSTX Facility Design Capability and Technology Challenges}

The NSTX facility is designed to achieve the NSTX mission with the following capabilities:

- $\mathrm{I}_{\mathrm{p}}=1 \mathrm{MA}$ for low collisionality at relevant densities,

- $\mathrm{R} / \mathrm{a} \geq 1.26$, including $\mathrm{OH}$ solenoid and coaxial helicity injection ${ }^{7}$ (CHI) for startup, 
- High Harmonic Fast Wave ${ }^{8}$ (HHFW - 6 MW, 5 sec), Coaxial Helicity Injection (CHI), Neutral Beam Injection (NBI - $5 \mathrm{MW}, 5 \mathrm{sec}$ ) for heating and $\mathrm{j}(\mathrm{r})$ control,

- Close-fitting conducting shell for maximum $\beta$ and $\beta_{\mathrm{N}}$, and

- Pulse length $5 \mathrm{sec} \geq$ skin time $\approx \mathrm{L} / \mathrm{R}$ time.

The NSTX device design is shown in Fig. 1. The device center-stack is designed and fabricated to allow for the very low aspect ratio $\mathrm{R} / \mathrm{a} \geq 1.26$ operation. It has a sufficient ohmic drive to create $1 \mathrm{MA}$ ohmically heated discharges. The center stack is connected to the outer vessel via ceramic insulators and bellows to provide an electrical isolation for $\mathrm{CHI}$ and a mechanical isolation to allow for the relative growth of center-stack with respect to the outer vacuum vessel during bakeout and operation. The device is designed with close-fitted $1.2 \mathrm{~cm}$ thick copper passive stabilizing plates for MHD mode stabilization. The CHI and HHFW will be used for the initial plasma start-up studies while ECH + HHFW is considered for RF only start-up as an upgrade. The NBI heating and current drive system is also expected to provide plasma rotation for mode stabilization as well as central plasma fueling. The NBI system will be also used for the NBI based plasma profile diagnostics such as CHERS (both toroidal and poloidal) and MSE (Motional Stark Effect) diagnostics. Presently, the NSTX device is being assembled in the NSTX Test Cell at PPPL, and the first plasma is planned for early 1999. The TFTR power supplies were reconfigured for NSTX. The device assembly and all the subsystems needed for the first plasma are scheduled to be completed by Dec. 1998, and the integrated systems test will commence in Jan. 1999. The HHFW antenna components are being fabricated and an innovative design was developed for the 12 element HHFW antenna decoupler / matching system. The HHFW system and the multi-pulse Thomson scattering system will become operational in the summer of 1999. The NBI heating system and associated NBI based diagnostics such as the CHERS will be commissioned in the summer of year 2000 .
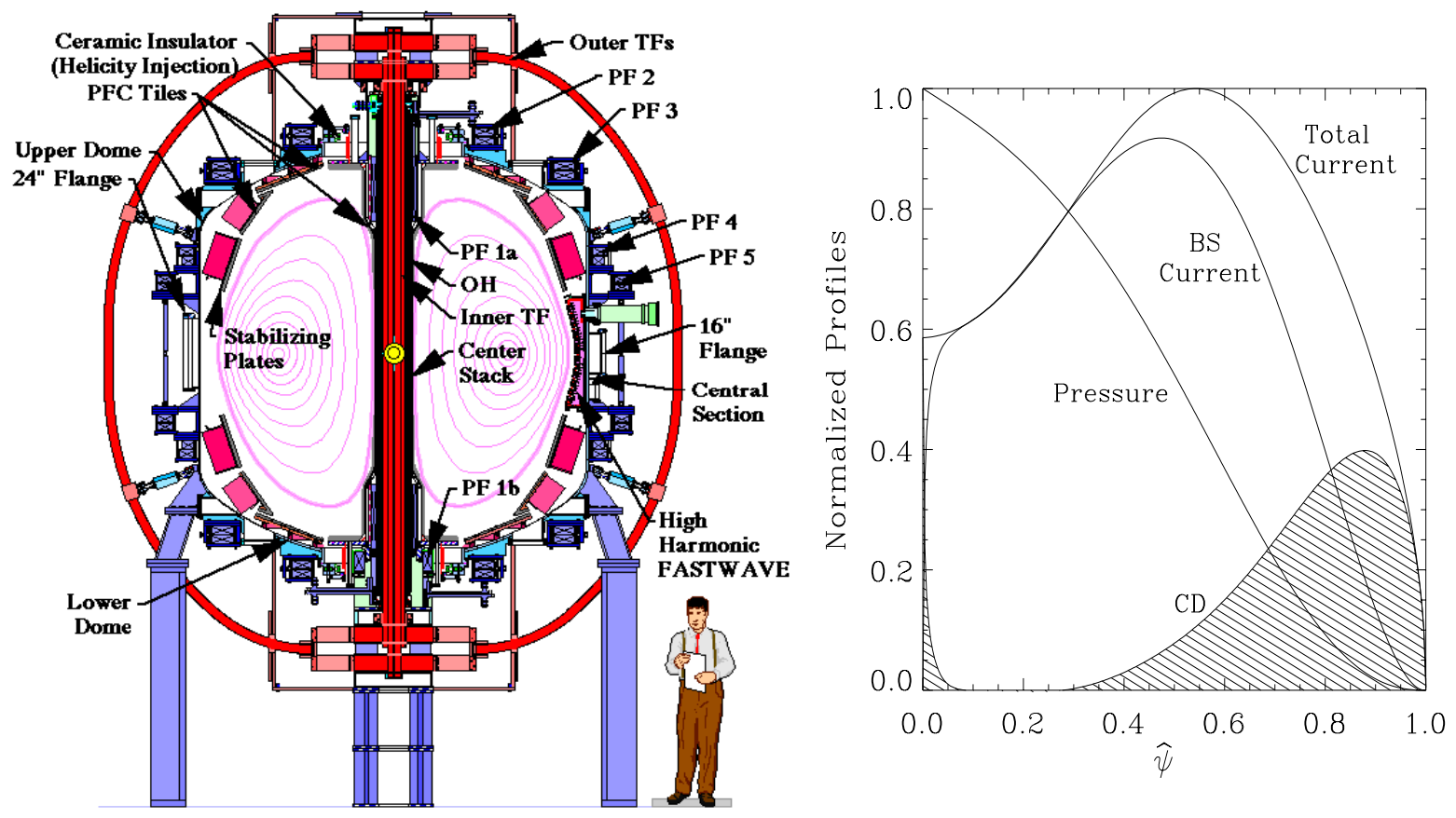

Fig. 1. NSTX Device Cross Section

Fig. 2. NSTX Adv. ST Regime: $\beta_{T}=40 \%$, $\beta_{N}=8.5, q_{95}=14, f_{b}=0.77, \kappa=2$, and $\delta=0.45$.

\section{Access to Advanced ST Regimes}

The ultimate goal of the NSTX research program is to access the advanced ST regime with simultaneous high beta, high bootstrap fraction, and high confinement regime in non-transient fashion. The ST configuration, due to the short outboard connection length combined with strong global 
magnetic shear, and the naturally high $\kappa$ and $\delta$, has the potential of achieving a high-performance regime with high plasma $\beta$ and $\mathrm{f}_{\mathrm{bs}}$ approaching unity. The predicted MHD stability limit against low $\mathrm{n}$-kinks and high-n ballooning modes is very high: $\beta_{\mathrm{T}} \rightarrow 60 \%, \beta_{\mathrm{N}} \rightarrow 8$ with $\mathrm{f}_{\mathrm{bs}} \approx 100 \%$ for $\kappa \approx 3.4$. In this regime, a close-fitting conducting shell with $r_{w a l l} / a \leq 1.2$ is needed for suppressing the low-n kink modes. For $\kappa \approx 2$ as planned for NSTX in the initial configuration, an ideal MHD stable regime with $\beta_{\mathrm{T}} \approx 40 \%, \beta_{\mathrm{N}} \approx 8$ with $\mathrm{f}_{\mathrm{bs}} \approx 75 \%$ is predicted. In Fig. 2 , the corresponding plasma current profile are shown. The $\mathrm{j}_{\mathrm{bs}}$ is indeed relatively well-aligned with the $\mathrm{j}_{\text {Total }}$, and an outer region current drive contribution of $\approx 20 \%$ is required. NSTX has a sufficient heating power to reach the desired $\beta$ value $(\approx 40 \%)$ with a relatively modest confinement assumption of $\mathrm{H}$-factor of $\approx 2$. A plasma pulse length of $5 \mathrm{sec}$ is sufficient to allow the current profile j(r) to fully relax. The low-n kinks are predicted to be stabilized by a close fitting conducting wall together with plasma rotation induced by NBI. For the j(r) control, the combination of NBI, HHFW, and CHI systems will be used to augment the bootstrap current. The TRANSP calculations show that NBI is capable of driving 100-200 kA of current in the central region which should be sufficient to provide the small central seed current $(\approx \mathrm{a}$ few $\mathrm{kA})$ required. For off-axis current drive, a twelve-element real-time-phased HHFW antenna array will be used for driving up to $300 \mathrm{kA}$ of off-axis current to supplement the bootstrap current. Theoretical analyses and modeling calculations show that the HHFW power absorption is one to two orders of magnitude larger in the NSTX parameters than conventional aspect ratio tokamaks. The strong singlepass absorption together with the real-time antenna phasing capability allows efficient off-axis current drive by HHFW. As for the edge current drive, the CHI is the most promising tool. The expected edge current for CHI in the well formed ST may be estimated as $\mathrm{I}_{\text {inj }} \mathrm{x}_{\mathrm{C}} \mathrm{q}_{95}$ where $\mathrm{I}_{\text {inj }}$ is the current injected into the plasma by CHI. For NSTX, up to $350 \mathrm{kA}$ of edge current may be driven by CHI with injection of $\approx 25 \mathrm{kA}$ for the expected $\mathrm{q}_{95} \approx 14$. It should be noted that the ideal ballooning mode prediction may turn out to be conservative for NSTX, because of the strong FLR and trapped particle effects in the ST configuration. If true, the higher edge pressure gradients in NSTX will further reduce the edge $\mathrm{CD}$ requirement. Another obvious path if the edge current drive turned out to be not practical is to move toward a higher elongation regime where the bootstrap current can be near $100 \%$ with very high beta. Recent ideal MHD calculations indicate that the details of the plasma boundary shape in the low field region is quite important for the ideal ballooning stability. The NSTX device poloidal field coils are configured therefore to give sufficient outer boundary shape control capability.
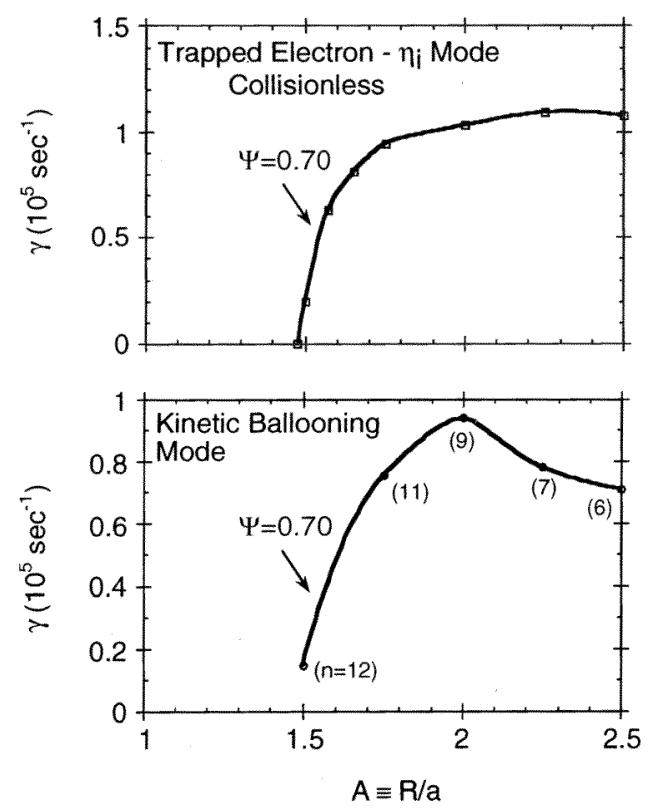

Fig. 3 Electrostatic and Electromagnetic Instability Suppression for low R/a.

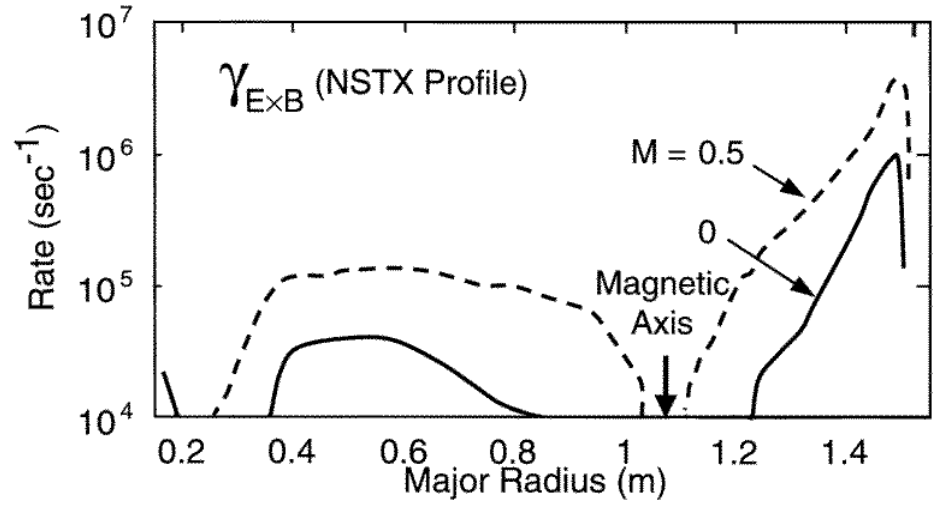

Fig. 4. Expected sheared flow rate profiles in NSTX 
IAEA-CN-69/ICP/01(R)-EX4/1(R)

\section{Prospect of High Confinement}

The ST configuration has the prospect of achieving very high confinement in the advanced ST regime. The predicted linear growth rates of kinetic, electrostatic mode such as dissipated trapped particle and $\eta$-i modes and electromagnetic mode such as kinetic ballooning microturbulence are found to be greatly reduced due to favorable drift orbits as R/a falls below 1.5 as shown by Fig. 3. 9 These properties are expected to improve plasma confinement in the outer region of NSTX. Due to the high beta nature of the NSTX plasmas, a strong plasma sheared flow rate, $\gamma_{\operatorname{ExB}}(\mathrm{sec}-1)$, over a significant portion of the minor radius, is predicted - particularly with NBI toroidal drive (toroidal Mach number $M=0.5$ ) as shown in Fig. 4. If the flow shear rate (and associated radial electric field shear rate) is sufficiently high compared to the turbulence growth rate, then it could lead to the turbulence suppression leading to reduced transport and improved plasma confinement. As can be seen from the Figs. 3 and 4, the expected shearing rate could readily exceed the linear growth rates of expected turbulence in the ST regime. ${ }^{10}$ In addition, due to the high beta as well as high poloidal field nature of the ST configuration, a significant absolute magnetic well $(\leq 30 \%)$ is expected to form in the core region, which tends to stabilize a class of plasma instabilities. This absolute minimum B formation and its effect on plasma confinement is an interesting area of research for NSTX.

\section{Non-Inductive Start-up and Power and Particle Handling}

In order to eliminate the $\mathrm{OH}$ solenoid, it is important to develop an efficient non-inductive startup tool for ST. The relatively modest magnetic flux and helicity per plasma current for ST tend to ease noninductive startup requirements. The main tool for NSTX is the coaxial-helicity-injection (CHI). The NSTX device is designed with the ceramic electrical insulation breaks located at both ends of the center-stack which would permit dc-biasing (up to $2 \mathrm{kV}$ ) between the center-stack and outer vacuum chamber. The CHI power supply is $2 \mathrm{kV}-50 \mathrm{kA}$ rectifier which should be sufficient to produce $500 \mathrm{kA}$ of non-inductive ST discharges from zero current. A TSC simulation shows that the HHFW heating and current drive (CD) along with bootstrap current (and a small amount of $\mathrm{OH}$ assist) can ramp up the current to full value of $1 \mathrm{MA}$. In addition, a start-up experimental plan based only on RF using the ECH start-up ${ }^{11}$ together with HHFW current ramp-up is also under study.

Due to inherently high power density of ST, it is essential to develop an acceptable power and particle handling concept. The ST plasmas permit inboard limited plasmas with mostly diverted SOL (the so-called natural divertor configuration), large magnetic variations along the SOL field line (e.g., strong magnetic mirror ratios up to 4-to-1 and field line curvatures), and potentially large flux tube expansion (an order of 10) and strong interchange instabilities due to high edge pressure gradients. These edge properties may lead to an effective power and particle handling concept for STs.

\section{REFERENCES:}

[1] PENG, Y-K. M., STRICKLER, D. J., Nuclear Fusion 26, 576(1986).

[2] CHENG, E. T., et al., Fusion Engineering and Design 38, 219 (1998).

[3] See for example, NADJMABADI, F., et al., and ROBINSON, D. C., et al., at this conference.

[4] GATES, D., et al., Physics of Plasmas, May (1998) and GRYAZNEVICH, M., et al., EPS Proceedings (1998).

[5] KAYE, S., et al., Bull Am. Phys. Soc. 42 (1997).

[6] MENARD, J., et al., Nucl. Fusion 37, 595 (1997).

[7] JARBOE, T., et al., Plasma Phys. Contr. Nuclear Fusion Research 1994, 1, 725 (IAEA, 1995).

[8] ONO, M., Physics of Plasmas 2, 4075 (1995).

[9] REWOLDT, G., et al., Physics of Plasmas 3, 1667 (1996).

[10] STAMBAUGH, R., et al., Plasma Phys. Contr. Nuclear Fusion Research 1996, 3, 395 (IAEA, 1997).

[11] FOREST, C., et al., Phys. Rev. Letters 68, 3559 (1992). 